\title{
Existence of longitudinal waves in pre-stressed anisotropic elastic medium
}

\author{
NeEtu GARG \\ Department of Applied Mathematics, University Institute of Engineering 8 Technology, \\ Kurukshetra University, Kurukshetra 136 119, India. \\ e-mail: ng_99@rediffmail.com
}

In a pre-stressed anisotropic elastic medium, three types of quasi-waves propagate along an arbitrary direction. In general, none of the waves is truly longitudinal. The present study finds the specific directions in a pre-stressed anisotropic elastic medium along which longitudinal waves may propagate. This paper demonstrates how the propagation of longitudinal waves is affected by various pre-stresses present in the medium. The study establishes the explicit expressions defining the existence and propagation of longitudinal waves in pre-stressed anisotropic elastic medium. These expressions involve not only the direction and elastic stiffness of the medium, but also the prestresses present in the medium. Changes in conditions for the existence of longitudinal waves in orthotropic, monoclinic and triclinic anisotropies are discussed in detail. The most important part of the paper is a practical aspect suggested to calculate the specific directions for the existence of longitudinal waves in pre-stressed anisotropic elastic medium. In this approach, only those parameters are used that can be observed by the receiver in a geophysical experiment of wave propagation. The existence of longitudinal waves has been shown graphically using a numerical example for three types of anisotropic symmetries in elastic medium.

\section{Introduction}

Wave propagation in pre-stressed anisotropic elastic materials finds its importance in geophysics and other branches of applied sciences such as petroleum engineering, electronics, electrical engineering and seismology. Anisotropy is the property that a solid body has, when its elastic properties vary with direction. This subject has received a lot of attention, mostly because the anisotropy may directly relate to the deformation in the mantle and stresses in the earth's crust, and therefore provides information to put constraints on tectonic and geodynamic processes (Crampin 1987). Due to the variations of temperature, slow process of creep and gravitational field, etc., a considerable amount of stress, called pre-stress, is stored in the layers of the earth (Biot 1940). These stresses modify the elastic properties of the layers and, hence affect the propagation of waves there (Biot 1965). In the past, a number of researchers (Cauchy 1827; Biot 1939, 1940, 1965; Birch 1947, 1952; Truesdell 1952; Hayes 1963; Tolstoy 1982; Man and Lu 1987; Degtyar and Rokhlin 1998; Prikazchikov and Rogerson 2003; Sharma and Garg 2006) have considered various problems on wave propagation in pre-stressed elastic materials.

In a pre-stressed unbounded anisotropic elastic medium, three types of waves propagate along an arbitrary (general) direction (Sharma and Garg 2006). Associated with each direction, there are three independent waves. Particle displacements due to these waves form a mutually orthogonal set. In general, none of the three displacement vectors coincide with the phase direction, i.e., none of the waves is truly longitudinal. Longitudinal wave in an anisotropic elastic medium is defined as the wave motion in which the particle motion (i.e., the

Keywords. General anisotropy; elastic stiffness; pre-stress; group velocity; ray direction; longitudinal waves; polarization. 
polarization vector) is in the direction of the propagation of plane wave. The problem of existence of longitudinal waves in anisotropic elastic medium has been considered by many researchers. Borgnis (1955) computed the direction along which a longitudinal wave can propagate in anisotropic elastic materials for trigonal, hexagonal, tetragonal and cubic symmetries. Aleksandrove (1956) extended the computation of such a specific propagation direction to orthotropic materials. The existence of a longitudinal wave in an anisotropic elastic material was proved by Truesdell (1966); Kolodner (1996) and Shuvalov (1998). The most elaborated available work on this topic is a paper by Ting (2006). In this paper, the expressions are derived to explain the phase direction along which a longitudinal wave can propagate in anisotropic medium.

In the present work, the effect of prestresses on the existence of longitudinal waves in anisotropic elastic medium is taken into consideration. Due to pre-stresses, medium of any type (isotropic/anisotropic) behaves anisotropic to wave propagation, and equation of motion is significantly affected. Any condition on elastic properties of a medium doesn't confine only to elastic constants, but also affects the structure of prestresses. Thus, the specific directions involving elastic properties of the medium as well as the pre-stresses, for the existence of longitudinal waves in a pre-stressed anisotropic elastic medium are determined here. Moreover, it is the group velocity and the particle motion that are recorded at an observatory. Hence, at the observation point, the particle motion can only be compared with ray direction. Keeping this in mind, the problem is also discussed taking these two parameters into consideration. It is shown that the relations obtained in this case may not be solved directly for an analytical or numerical solution. However, the verifications can be made graphically for any given numerical model of a pre-stressed medium. The specific directions for the existence of longitudinal waves are shown graphically using numerical examples for three types of anisotropies (triclinic, monoclinic and orthorhombic).

\section{Longitudinal waves in a pre-stressed anisotropic elastic medium}

Consider the wave motion in a general anisotropic elastic medium under homogeneous pre-stresses $S_{i j}(i, j=1,2,3)$, in the absence of body force. The medium is represented by the elastic tensor $B_{i j k l}$ and density $\rho$. A non-symmetric square matrix of order six, $\mathbf{B}=\left\{b_{i j}\right\}$, is used to denote the elastic properties of the medium in two-suffix notations.
Let a unit vector $\mathbf{N}=\left(n_{1}, n_{2}, n_{3}\right)$, define the phase direction. The Christoffel equations governing the wave motion in the medium (Sharma and Garg 2006), are written as:

$$
\left(X_{i k}-h \delta_{i k}\right) U_{k}=0, \quad(i=1,2,3),
$$

where $\delta_{i j}$ is the Kronecker delta, and $U_{k}$ are the Cartesian components of displacement amplitude vector $\mathbf{U}$.

Here,

$$
h=v^{2}-\mathbf{N S N}^{T} / \rho,
$$

and, $X_{i k}$ are the elements of a matrix

$$
\mathbf{X}=\frac{\left[\mathbf{Z}-\mathbf{S}+\mathbf{S N}^{T} \mathbf{N}-\mathbf{N}^{T} \mathbf{N S}\right]}{\rho}
$$

where $\mathbf{N}^{T}$ is the transpose of $\mathbf{N}$, and the matrix $\mathbf{S}$ is given as:

$$
\mathbf{S}=\frac{1}{2}\left\{S_{i j}\right\}
$$

In equation (2), $v$ is the phase velocity and in equation (3), the square matrix $\mathbf{Z}$ of order three is defined through its elements given by:

$$
\begin{array}{ll}
Z_{11}=\mathbf{N} \mathbf{A}_{1} \mathbf{N}^{T}, & Z_{22}=\mathbf{N} \mathbf{A}_{2} \mathbf{N}^{T}, \\
Z_{33}=\mathbf{N A}_{3} \mathbf{N}^{T}, & Z_{12}=\mathbf{N} \mathbf{A}_{4} \mathbf{N}^{T}, \\
Z_{13}=\mathbf{N A}_{5} \mathbf{N}^{T}, & Z_{21}=\mathbf{N} \mathbf{A}_{6} \mathbf{N}^{T}, \\
Z_{23}=\mathbf{N A}_{7} \mathbf{N}^{T}, & Z_{31}=\mathbf{N} \mathbf{A}_{8} \mathbf{N}^{T}, \\
Z_{32}=\mathbf{N A}_{9} \mathbf{N}^{T}, &
\end{array}
$$

where $\mathbf{A}_{\mathbf{j}}(\mathbf{j}=1,2, \ldots, 9)$, the square matrices of order three, are defined as follows:

$$
\begin{aligned}
& \mathbf{A}_{1}=\left\{b_{11}, b_{16}, b_{15} ; b_{61}, b_{66}, b_{65} ; b_{51}, b_{56}, b_{55}\right\} ; \\
& \mathbf{A}_{2}=\left\{b_{66}, b_{62}, b_{64} ; b_{26}, b_{22}, b_{24} ; b_{46}, b_{42}, b_{44}\right\} ; \\
& \mathbf{A}_{3}=\left\{b_{55}, b_{54}, b_{53} ; b_{45}, b_{44}, b_{43} ; b_{35}, b_{34}, b_{33}\right\} ; \\
& \mathbf{A}_{4}=\left\{b_{16}, b_{12}, b_{14} ; b_{66}, b_{62}, b_{64} ; b_{56}, b_{52}, b_{54}\right\} ; \\
& \mathbf{A}_{5}=\left\{b_{15}, b_{14}, b_{13} ; b_{65}, b_{64}, b_{63} ; b_{55}, b_{54}, b_{53}\right\} ; \\
& \mathbf{A}_{6}=\left\{b_{61}, b_{66}, b_{65} ; b_{21}, b_{26}, b_{25} ; b_{41}, b_{46}, b_{45}\right\} ;
\end{aligned}
$$




$$
\begin{aligned}
& \mathbf{A}_{7}=\left\{b_{65}, b_{64}, b_{63} ; b_{25}, b_{24}, b_{23} ; b_{45}, b_{44}, b_{43}\right\} ; \\
& \mathbf{A}_{8}=\left\{b_{51}, b_{56}, b_{55} ; b_{41}, b_{46}, b_{45} ; b_{31}, b_{36}, b_{35}\right\} ; \\
& \mathbf{A}_{9}=\left\{b_{56}, b_{52}, b_{54} ; b_{46}, b_{42}, b_{44} ; b_{36}, b_{32}, b_{34}\right\} .
\end{aligned}
$$

The direction of $\mathbf{U}$ defines the polarization of the concerned wave. So, for longitudinal wave, equation (1) implies:

$$
\left(X_{i k}-h \delta_{i k}\right) n_{k}=0 .
$$

Using the values of $X_{i k}$ and $h$, this last equation gives three non-homogeneous equations, given as:

$$
\begin{aligned}
\mathbf{N} & \left.\mathbf{A}_{1} n_{1}+\mathbf{A}_{4} n_{2}+\mathbf{A}_{5} n_{3}\right) \mathbf{N}^{T} \\
& -n_{1}^{2}\left(\frac{S_{11}}{2} n_{1}+S_{12} n_{2}+S_{13} n_{3}\right) \\
& -n_{1}\left(\frac{S_{22}}{2} n_{2}^{2}+\frac{S_{33}}{2} n_{3}^{2}\right)-S_{23} n_{1} n_{2} n_{3} \\
& =\left(\rho v^{2}-\mathbf{N S N}^{T}\right) n_{1}, \\
\mathbf{N} & \left(\mathbf{A}_{6} n_{1}+\mathbf{A}_{2} n_{2}+\mathbf{A}_{7} n_{3}\right) \mathbf{N}^{T} \\
& -n_{2}^{2}\left(S_{12} n_{1}+\frac{S_{22}}{2} n_{2}+S_{23} n_{3}\right) \\
& -n_{2}\left(\frac{S_{11}}{2} n_{1}^{2}+\frac{S_{33}}{2} n_{3}^{2}\right)-S_{13} n_{1} n_{2} n_{3} \\
& =\left(\rho v^{2}-\mathbf{N S N}^{T}\right) n_{2}, \\
\mathbf{N} & \left(\mathbf{A}_{8} n_{1}+\mathbf{A}_{9} n_{2}+\mathbf{A}_{3} n_{3}\right) \mathbf{N}^{T} \\
& -n_{3}^{2}\left(S_{13} n_{1}+S_{23} n_{2}+\frac{S_{33}}{2} n_{3}\right) \\
& -n_{3}\left(\frac{S_{11}}{2} n_{1}^{2}+\frac{S_{22}}{2} n_{2}^{2}\right)-S_{12} n_{1} n_{2} n_{3} \\
& =\left(\rho v^{2}-\mathbf{N S N}^{T}\right) n_{3} .
\end{aligned}
$$

Multiplying equations (8)-(10) by $n_{1}, n_{2}$ and $n_{3}$, respectively, and adding together yields:

$$
\begin{aligned}
\rho v^{2}= & b_{11} n_{1}^{4}+b_{22} n_{2}^{4}+b_{33} n_{3}^{4} \\
& +2\left(\gamma_{1} n_{2}^{2} n_{3}^{2}+\gamma_{2} n_{3}^{2} n_{1}^{2}+\gamma_{3} n_{1}^{2} n_{2}^{2}\right) \\
& +4 n_{2} n_{3}\left(\tilde{b}_{14} n_{1}^{2}+\tilde{b}_{24} n_{2}^{2}+\tilde{b}_{34} n_{3}^{2}\right)
\end{aligned}
$$

$$
\begin{aligned}
& +4 n_{3} n_{1}\left(\tilde{b}_{15} n_{1}^{2}+\tilde{b}_{25} n_{2}^{2}+\tilde{b}_{35} n_{3}^{2}\right) \\
& +4 n_{1} n_{2}\left(\tilde{b}_{16} n_{1}^{2}+\tilde{b}_{26} n_{2}^{2}+\tilde{b}_{36} n_{3}^{2}\right) .
\end{aligned}
$$

The coefficients in (11) involve the pre-stress components and are defined as follows:

$$
\begin{aligned}
& \gamma_{1}=2 b_{44}+b_{23}+\frac{S_{22}-S_{33}}{2}, \\
& \gamma_{2}=2 b_{55}+b_{13}+\frac{S_{11}-S_{33}}{2}, \\
& \gamma_{3}=2 b_{66}+b_{12}+\frac{S_{11}-S_{22}}{2}, \\
& \tilde{b}_{14}=2 b_{56}+b_{14}-\frac{S_{23}}{2}, \\
& \tilde{b}_{24}=b_{24}-\frac{S_{23}}{2}, \quad \tilde{b}_{34}=b_{34}-\frac{S_{23}}{2}, \\
& \tilde{b}_{15}=b_{15}-\frac{S_{13}}{2}, \quad \tilde{b}_{25}=2 b_{46}+b_{25}-\frac{S_{13}}{2}, \\
& \tilde{b}_{35}=b_{35}-\frac{S_{13}}{2}, \quad \tilde{b}_{16}=b_{16}-\frac{S_{12}}{2}, \\
& \tilde{b}_{26}=b_{26}-\frac{S_{12}}{2}, \quad \tilde{b}_{36}=2 b_{45}+b_{36}-\frac{S_{12}}{2} .
\end{aligned}
$$

Alternatively, equation (1) can be written as:

$$
\mathbf{Y}(\mathbf{N}) \mathbf{U}=\rho v^{2} \mathbf{U},
$$

where, the acoustic tensor $\mathbf{Y}(\mathbf{N})$ is expressed as:

$$
Y_{i k}(\mathbf{N})=b_{i j k l} n_{j} n_{l} .
$$

For a non-trivial solution of the polarization vector $\mathbf{U}$, we must have:

$$
\operatorname{det}\left(\mathbf{Y}(\mathbf{N})-\rho v^{2} \mathbf{I}\right)=0
$$

in which $\mathbf{I}$ is the identity tensor. The phase velocity $v$ is obtained from equation (13) as:

$$
\rho v^{2}=b_{i j k l} U_{i} n_{j} U_{k} n_{l},
$$

where eigen vector $\mathbf{U}$ in (13) is normalized to represent polarization.

So, for a longitudinal wave:

$$
\mathbf{U}=\mathbf{N}
$$


Then the equations (14) and (16) give

$$
\begin{aligned}
& b_{i j k l} n_{j} n_{l} n_{k}=\rho v^{2} n_{i}, \\
& \rho v^{2}=b_{i j k l} n_{i} n_{j} n_{k} n_{l} .
\end{aligned}
$$

Referred to a new co-ordinate system $x_{i}^{*}(i=1,2,3)$, the elastic compliance $b_{1111}^{*}$ is expressed as:

$$
b_{1111}^{*}=b_{i j k l} n_{i} n_{j} n_{k} n_{l}=b_{11}(\mathbf{N})
$$

where $\mathbf{N}$ is a unit vector along the $x_{1}^{*}$-axis. From (11) and (19)

$$
\begin{aligned}
b_{11}(\mathbf{N})= & b_{11} n_{1}^{4}+b_{22} n_{2}^{4}+b_{33} n_{3}^{4} \\
& +2\left(\gamma_{1} n_{2}^{2} n_{3}^{2}+\gamma_{2} n_{3}^{2} n_{1}^{2}+\gamma_{3} n_{1}^{2} n_{2}^{2}\right) \\
& +4 n_{2} n_{3}\left(\tilde{b}_{14} n_{1}^{2}+\tilde{b}_{24} n_{2}^{2}+\tilde{b}_{34} n_{3}^{2}\right) \\
& +4 n_{3} n_{1}\left(\tilde{b}_{15} n_{1}^{2}+\tilde{b}_{25} n_{2}^{2}+\tilde{b}_{35} n_{3}^{2}\right) \\
& +4 n_{1} n_{2}\left(\tilde{b}_{16} n_{1}^{2}+\tilde{b}_{26} n_{2}^{2}+\tilde{b}_{36} n_{3}^{2}\right) .
\end{aligned}
$$

Note that instead of 21 elastic constants and 6 pre-stress components, the expression (21) for $b_{11}(\mathbf{N})$ depends only on 15 elastic coefficients in terms of $b_{i j}$ and $S_{i j}$. Following Ting (2006), the necessary and sufficient condition for a longitudinal wave to propagate is to find the direction $\mathbf{N}$ at which $b_{11}(\mathbf{N})$ is a stationary value. Moreover, from $(21), b_{11}(\mathbf{N})$ is stationary when $v$ becomes independent of $\mathbf{N}$. It means no spatial dispersion for wave propagation. This implies that group velocity for the propagation of energy will be equal to $v(\mathbf{N})$.

\section{Longitudinal waves in orthotropic materials}

A material having three mutually perpendicular planes of elastic symmetry at a point is said to have orthotropic symmetry. In this section, the aim is to find out the direction $\mathbf{N}$ for which a longitudinal wave can propagate in an orthotropic material. The material represented by elastic matrix $\left\{b_{I J}\right\}$ behaves orthotropic when some of the elastic parameters vanish as given below.

$$
\begin{aligned}
\tilde{b}_{14} & =\tilde{b}_{15}=\tilde{b}_{16}=\tilde{b}_{24}=\tilde{b}_{25}=\tilde{b}_{26} \\
& =\tilde{b}_{34}=\tilde{b}_{35}=\tilde{b}_{36}=0 ; \\
b_{45} & =b_{46}=b_{56}=0 .
\end{aligned}
$$

The pre-stress is defined by normal components only and

$$
S_{23}=S_{13}=S_{12}=0 .
$$

Thus, in an orthotropic medium, equation (21) takes the form,

$$
\begin{aligned}
b_{11}(\mathbf{N})= & b_{11} n_{1}^{4}+b_{22} n_{2}^{4}+b_{33} n_{3}^{4} \\
& +2\left(\gamma_{1} n_{2}^{2} n_{3}^{2}+\gamma_{2} n_{3}^{2} n_{1}^{2}+\gamma_{3} n_{1}^{2} n_{2}^{2}\right),
\end{aligned}
$$

where the coefficients depending upon pre-stress components are $\gamma_{j}(j=1,2,3)$ only. The $b_{11}(\mathbf{N})$ shown in above equation depends on six elastic coefficients involving normal components of pre-stress instead of nine elastic coefficients and three normal components of pre-stress. Since the right-hand side of above equation is a function of $n_{1}^{2}, n_{2}^{2}, n_{3}^{2}$, so components of $\mathbf{N}$ should be considered as non-negative.

To find the extremum of $b_{11}(\mathbf{N})$ subject to the condition $n_{i} n_{i}=1$, define a function:

$$
\begin{gathered}
f(\mathbf{N}, \lambda)=b_{11}(\mathbf{N})-2 \lambda\left(n_{i} n_{i}-1\right), \\
(i=1,2,3),
\end{gathered}
$$

where $-2 \lambda$ is a non-zero Lagrange's undetermined multiplier. Vanishing of partial derivatives, given by:

$$
\begin{aligned}
& \frac{\partial f}{\partial \lambda}=0, \quad \frac{\partial f}{\partial n_{1}}=0, \\
& \frac{\partial f}{\partial n_{2}}=0, \quad \frac{\partial f}{\partial n_{3}}=0,
\end{aligned}
$$

yield the following four equations,

$$
\begin{aligned}
& n_{1}^{2}+n_{2}^{2}+n_{3}^{2}=1, \\
& n_{1}\left(b_{11} n_{1}^{2}+\gamma_{3} n_{2}^{2}+\gamma_{2} n_{3}^{2}-\lambda\right)=0, \\
& n_{2}\left(\gamma_{3} n_{1}^{2}+b_{22} n_{2}^{2}+\gamma_{1} n_{3}^{2}-\lambda\right)=0, \\
& n_{3}\left(\gamma_{2} n_{1}^{2}+\gamma_{1} n_{2}^{2}+b_{22} n_{3}^{2}-\lambda\right)=0 .
\end{aligned}
$$

Multiplying equations (27), (28) and (29) by $n_{1}$, $n_{2}$ and $n_{3}$, respectively, and adding together, we 
have from (24),

$$
\lambda=b_{11}(\mathbf{N}) .
$$

So, our aim is to find out $\mathbf{N}$ and $\lambda$ that satisfy equations (26)-(30), for which we will take into consideration some special cases one by one.

Case (1): $N$ is along the $x_{1}$-axis. Then, the results are:

$$
\begin{aligned}
& n_{1}=1, \quad n_{2}=n_{3}=0, \quad \lambda=b_{11}, \\
& b_{11}(\mathbf{N})=b_{11}=\rho v^{2} .
\end{aligned}
$$

Therefore,

$$
v=\sqrt{\frac{b_{11}}{\rho}} .
$$

This implies that, in orthotropic medium, a longitudinal wave propagates along $x_{1}$-axis. The wave propagates with velocity $\sqrt{b_{11} / \rho}$ and is not affected by the presence of pre-stresses in the medium. The same situation holds when $\mathbf{N}$ is along the $x_{2}$-axis and $x_{3}$-axis.

Case (2): $\mathbf{N}$ is on a co-ordinate plane. For $n_{1}=0$, equations (26)-(29) give:

$$
\begin{aligned}
& n_{2}^{2}=\frac{b_{33}-\gamma_{1}}{\left(b_{22}-\gamma_{1}\right)+\left(b_{33}-\gamma_{1}\right)}, \\
& n_{3}^{2}=\frac{b_{22}-\gamma_{1}}{\left(b_{22}-\gamma_{1}\right)+\left(b_{33}-\gamma_{1}\right)},
\end{aligned}
$$

and equation (24) has the expression:

$$
b_{11}(\mathbf{N})=\frac{b_{22} b_{33}-\gamma_{1}^{2}}{\left(b_{22}-\gamma_{1}\right)+\left(b_{33}-\gamma_{1}\right)}=b_{1}^{*}, \text { (say), }
$$

where $\gamma_{1}$ is as defined in (12). Thus, $b_{1}^{*}$ involves $S_{22}$ and $S_{33}$. In (32), $n_{2}^{2}$ and $n_{3}^{2}$ are positive. This means, $b_{22}-\gamma_{1}$ and $b_{33}-\gamma_{1}$ should be of the same sign. If $\gamma_{1}$ lies between $b_{22}$ and $b_{33}$, then equation (32) will be impossible for any positive value of $n_{2}^{2}$ and $n_{3}^{2}$, and a longitudinal wave cannot propagate along $\mathbf{N}$ on the plane $x_{1}=0$. Thus, a longitudinal wave with phase direction $\left(0, n_{2}, n_{3}\right)$ satisfying the conditions (32) and (33) involving initial stress components, can propagate in the $x_{2} x_{3}$-plane. Note that these conditions are affected by the pre-stress components, $S_{22}$ and $S_{33}$. The results when $\mathbf{N}$ is on the plane $n_{2}=0$ and $n_{3}=0$ can be determined in a similar way as above.

Case (3): $\mathbf{N}$ is not on any coordinate plane. Then, equations (26)-(29) can be written as:

$$
\left[\begin{array}{cccc}
1 & 1 & 1 & 0 \\
b_{11} & \gamma_{3} & \gamma_{2} & 1 \\
\gamma_{3} & b_{22} & \gamma_{1} & 1 \\
\gamma_{2} & \gamma_{1} & b_{33} & 1
\end{array}\right]\left[\begin{array}{c}
n_{1}^{2} \\
n_{2}^{2} \\
n_{3}^{2} \\
-\lambda
\end{array}\right]=\left[\begin{array}{l}
1 \\
0 \\
0 \\
0
\end{array}\right]
$$

Using Cramer's rule,

$$
n_{i}^{2}=\frac{\alpha_{i}}{\Delta}, \quad(i=1,2,3),
$$

where $\Delta=\sum_{i} \alpha_{i}$.

Here,

$\alpha_{1}=\left(b_{22}-\gamma_{3}\right)\left(b_{33}-\gamma_{2}\right)-\left(\gamma_{1}-\gamma_{3}\right)\left(\gamma_{1}-\gamma_{2}\right)$.

The expressions for $\alpha_{2}\left(\alpha_{3}\right)$ can be obtained from that of $\alpha_{1}$, by interchanging the indices 1 and 2 (3).

Here,

$$
b_{11}(\mathbf{N})=\frac{\eta}{\Delta}=b_{\circ}^{*}, \quad(\text { say }),
$$

where,

$$
\begin{aligned}
\eta= & \frac{1}{3}\left[\left(\gamma_{1}+\gamma_{2}+\gamma_{3}\right) \Delta+\left(b_{11}-\gamma_{1}\right) \alpha_{1}\right. \\
& \left.+\left(b_{22}-\gamma_{2}\right) \alpha_{2}+\left(b_{33}-\gamma_{3}\right) \alpha_{3}\right] .
\end{aligned}
$$

Using (35) and (38), equation (37) can be written as:

$$
\begin{aligned}
b_{11}(\mathbf{N})= & \frac{1}{3}\left[\left(\gamma_{1}+\gamma_{2}+\gamma_{3}\right)+\left(b_{11}-\gamma_{1}\right) \eta_{1}^{2}\right. \\
& \left.+\left(b_{22}-\gamma_{2}\right) \eta_{2}^{2}+\left(b_{33}-\gamma_{3}\right) \eta_{3}^{2}\right]=b_{o}^{*},
\end{aligned}
$$

where $\gamma_{1}, \gamma_{2}$ and $\gamma_{3}$ are as defined in (12). Thus, $b_{\circ}^{*}$ involves $S_{11}, S_{22}$ and $S_{33}$. Since $n_{i}^{2}(i=1,2,3)$ are positive, so $\Delta$ and $\alpha_{i}(i=1,2,3)$ should be of same sign. Thus, in this case also, pre-stresses play an important role in determining the required condition for particle motion to be in phase direction. The derivation of (39) presented above is not valid when

$$
b_{11}=b_{22}=b_{33}=\gamma_{1}=\gamma_{2}=\gamma_{3} .
$$


When equation (40) holds, then the equation (24) gives $b_{11}(\mathbf{N})=b_{11}$, which does not depend upon any of $n_{i}$ or $S_{i i}(i=1,2,3)$. Hence, particle motion is always along any given phase direction when (40) holds.

In the absence of pre-stresses, i.e., taking $S_{11}=S_{22}=S_{33}=0$, all the expressions, derived in this section, will reduce to the results which are same as in Ting (2006).

\section{Triclinic materials}

A triclinic material is the most general anisotropic medium, and the elastic compliance $b_{11}(\mathbf{N})$ in this medium is as defined by equation (21). For stationary values of $b_{11}(\mathbf{N})$, proceeding as in section 3 ,

$$
\begin{aligned}
& n_{1}^{2}+n_{2}^{2}+n_{3}^{2}=1 \\
& n_{1}\left[b_{11} n_{1}^{2}+\gamma_{2} n_{3}^{2}+\gamma_{3} n_{2}^{2}+2 \tilde{b}_{14} n_{2} n_{3}\right. \\
& \left.\quad+3 \tilde{b}_{15} n_{1} n_{3}+3 \tilde{b}_{16} n_{1} n_{2}-\lambda\right] \\
& \quad+n_{3}\left[\tilde{b}_{25} n_{2}^{2}+\tilde{b}_{35} n_{3}^{2}\right]+n_{2}\left[\tilde{b}_{26} n_{2}^{2}+\tilde{b}_{36} n_{3}^{2}\right]=0
\end{aligned}
$$

$$
\begin{aligned}
& n_{2}\left[b_{22} n_{2}^{2}+\gamma_{1} n_{3}^{2}+\gamma_{3} n_{1}^{2}+2 \tilde{b}_{25} n_{1} n_{3}\right. \\
& \left.\quad+3 \tilde{b}_{24} n_{2} n_{3}+3 \tilde{b}_{26} n_{1} n_{2}-\lambda\right] \\
& \quad+n_{3}\left[\tilde{b}_{14} n_{1}^{2}+\tilde{b}_{34} n_{3}^{2}\right]+n_{1}\left[\tilde{b}_{16} n_{1}^{2}+\tilde{b}_{36} n_{3}^{2}\right]=0
\end{aligned}
$$

$$
\begin{aligned}
n_{3} & {\left[b_{33} n_{3}^{2}+\gamma_{1} n_{2}^{2}+\gamma_{2} n_{1}^{2}+2 \tilde{b}_{36} n_{1} n_{2}\right.} \\
& \left.+3 \tilde{b}_{34} n_{2} n_{3}+3 \tilde{b}_{35} n_{1} n_{3}-\lambda\right] \\
& +n_{2}\left[\tilde{b}_{14} n_{1}^{2}+\tilde{b}_{24} n_{2}^{2}\right]+n_{1}\left[\tilde{b}_{15} n_{1}^{2}+\tilde{b}_{25} n_{2}^{2}\right]=0 .
\end{aligned}
$$

Thus, in a triclinic medium, a longitudinal wave can propagate:

- along $x_{1}$-axis with $b_{11}(\mathbf{N})=b_{11}$, if

$$
b_{15}=b_{16}=0 \quad \text { and } \quad S_{12}=S_{13}=0 .
$$

Here, $b_{11}(\mathbf{N})$ doesn't involve any pre-stress component. Hence, longitudinal wave propagating along $x_{1}$-axis is not affected by the presence of pre-stress in the medium, provided condition (45) holds. The same result holds in the case of longitudinal wave propagating along $x_{2}$-axis and $x_{3}$-axis.

- along $\mathbf{N}$ on the co-ordinate plane $x_{1}=0$, if the following two coupled algebraic equations hold,

$$
\begin{aligned}
& \tilde{b}_{26} y^{3}+\tilde{b}_{25} y^{2}+\tilde{b}_{36} y+\tilde{b}_{35}=0, \\
& \tilde{b}_{24} y^{4}-\left(b_{22}-\gamma_{1}\right) y^{3}-3\left(\tilde{b}_{24}-\tilde{b}_{34}\right) y^{2} \\
& \quad+\left(b_{33}-\gamma_{1}\right) y-\tilde{b}_{34}=0,
\end{aligned}
$$

where $y=n_{2} / n_{3}$ and $\tilde{b}_{24}, \tilde{b}_{25}, \tilde{b}_{26}, \tilde{b}_{34}, \tilde{b}_{35}, \tilde{b}_{36}$ and $\gamma_{1}$ are as defined in (12). The above two equations involve all the pre-stress components except $S_{11}$. Thus, if these two equations have a real solution, then longitudinal wave can propagate in the $x_{2} x_{3}$-plane. The wave is affected by all the pre-stress components except $S_{11}$. Proceeding in a similar fashion, the conditions for propagation along $\mathbf{N}$ on the co-ordinate planes $x_{2}=0$ and $x_{3}=0$ can be established easily.

- along $\mathbf{N}$, which is not on any co-ordinate plane, if the following two equations are simultaneously true,

$$
\begin{aligned}
& \tilde{b}_{15} y_{1}^{2}\left(3-y_{1}^{2}\right)+\tilde{b}_{25} y_{2}^{2}\left(1-y_{1}^{2}\right)+\tilde{b}_{35}\left(1-3 y_{1}^{2}\right) \\
& \quad+y_{2}\left[\left(3 \tilde{b}_{16}-2 \tilde{b}_{36}\right) y_{1}^{2}+\tilde{b}_{26} y_{2}^{2}+\tilde{b}_{36}\right] \\
& \quad-y_{1} y_{2}\left[\tilde{b}_{14} y_{1}^{2}+\tilde{b}_{24} y_{2}^{2}+\left(3 \tilde{b}_{34}-2 \tilde{b}_{14}\right)\right] \\
& \quad+y_{1}\left[\left(b_{11}-\gamma_{2}\right) y_{1}^{2}+\left(\gamma_{3}-\gamma_{1}\right) y_{2}^{2}\right. \\
& \left.\quad-\left(b_{33}-\gamma_{2}\right)\right]=0 \\
& \quad \tilde{b}_{14} y_{1}^{2}\left(1-y_{2}^{2}\right)+\tilde{b}_{24} y_{2}^{2}\left(3-y_{2}^{2}\right)+\tilde{b}_{34}\left(1-3 y_{2}^{2}\right) \\
& \quad+y_{1}\left[\tilde{b}_{16} y_{1}^{2}+\left(3 \tilde{b}_{26}-2 \tilde{b}_{36}\right) y_{2}^{2}+\tilde{b}_{36}\right] \\
& \quad-y_{1} y_{2}\left[\tilde{b}_{15} y_{1}^{2}+\tilde{b}_{25} y_{2}^{2}+\left(3 \tilde{b}_{35}-2 \tilde{b}_{25}\right)\right] \\
& \quad+y_{2}\left[\left(\gamma_{3}-\gamma_{2}\right) y_{1}^{2}+\left(b_{22}-\gamma_{1}\right) y_{2}^{2}\right. \\
& \left.\quad-\left(b_{33}-\gamma_{1}\right)\right]=0
\end{aligned}
$$

where $y_{1}=n_{1} / n_{3}$ and $y_{2}=n_{2} / n_{3}$. Here, the coefficients $\tilde{b}_{14}, \tilde{b}_{24}, \tilde{b}_{34}, \tilde{b}_{15}, \tilde{b}_{25}, \tilde{b}_{35}, \tilde{b}_{16}, \tilde{b}_{26}, \tilde{b}_{36}$, $\gamma_{1}, \gamma_{2}$ and $\gamma_{3}$ involve with all the pre-stress components. Hence, longitudinal wave propagating, not in any of the co-ordinate plane, is affected by all the components of pre-stress present in the medium. Thus, in this case, all the components of pre-stress have their significant role in the propagation of longitudinal wave.

In the absence of pre-stress components, i.e., taking

$$
S_{i j}=0 \quad(i, j=1,2,3),
$$


all the expressions derived in this section will reduce to the results which are same as in Ting (2006).

\section{Monoclinic materials}

In monoclinic materials, there is only one plane of elastic symmetry. Without loss in generality, let the symmetry plane of the material be the co-ordinate plane $x_{3}=0$. This implies:

$$
b_{14}=b_{15}=b_{24}=b_{25}=b_{34}=b_{35}=b_{46}=b_{56}=0 .
$$

The elastic compliance $b_{11}(\mathbf{N})$ becomes,

$$
\begin{aligned}
b_{11}(\mathbf{N})= & b_{11} n_{1}^{4}+b_{22} n_{2}^{4}+b_{33} n_{3}^{4} \\
& +2\left(\gamma_{1} n_{2}^{2} n_{3}^{2}+\gamma_{2} n_{3}^{2} n_{1}^{2}+\gamma_{3} n_{1}^{2} n_{2}^{2}\right) \\
& +4 n_{1} n_{2}\left(\tilde{b}_{16} n_{1}^{2}+\tilde{b}_{26} n_{2}^{2}+\tilde{b}_{36} n_{3}^{2}\right) .
\end{aligned}
$$

Thus, $b_{11}(\mathbf{N})$ depends on nine elastic constants involving with four pre-stress components, $S_{11}, S_{22}$, $S_{33}$ and $S_{12}$. Proceeding as in section 4 , we can conclude that in a monoclinic medium, a longitudinal wave can propagate

- along $x_{1}$-axis with $b_{11}(\mathbf{N})=b_{11}$, if

$$
b_{16}=0 \quad \text { and } \quad S_{12}=0 .
$$

Therefore, if the medium is stressed with $S_{i i}(i=1,2,3)$ only, then longitudinal wave can propagate along $\mathrm{x}_{1}$-axis. The wave is not affected by these pre-stress components, $S_{11}, S_{22}$, and $S_{33}$ present in the medium. The results for propagation along $x_{2}$-axis holds in a same way as above.

- along $x_{3}$-axis always with

$$
b_{11}(\mathbf{N})=b_{33}
$$

without any other condition on elastic constants.

Thus, longitudinal wave can propagate along $x_{3}$-axis. The pre-stresses present in the medium has no effect on propagation of longitudinal waves propagating along $x_{3}$-axis.

- along $\mathbf{N}$ on the co-ordinate plane $x_{1}=0$, if $\gamma_{1}-b_{22}$ and $\gamma_{1}-b_{33}$ have the same sign and

$$
\tilde{b}_{26}\left(b_{33}-\gamma_{1}\right)+\tilde{b}_{36}\left(b_{22}-\gamma_{1}\right)=0,
$$

where $\tilde{b}_{26}, \tilde{b}_{36}$ and $\gamma_{1}$ are given as in equation (12). Here, the coefficients $\tilde{b}_{26}, \tilde{b}_{36}$ and $\gamma_{1}$ involve with the pre-stress components $S_{22}, S_{33}$ and $S_{12}$.
Thus, if longitudinal wave propagates in the $x_{2} x_{3}$-plane, then it is not affected by $S_{11}$. In the same way, the conditions for propagation along $\mathbf{N}$ on the co-ordinate plane $x_{2}=0$ and $x_{3}=0$ can be obtained.

- along $\mathbf{N}$ which is not on any co-ordinate plane, if the following two coupled cubic equations in $y_{1}$ and $y_{2}$ are simultaneously true,

$$
\begin{gathered}
y_{2}\left[\left(3 \tilde{b}_{16}-2 \tilde{b}_{36}\right) y_{1}^{2}+\tilde{b}_{26} y_{2}^{2}+\tilde{b}_{36}\right]+y_{1}\left[\left(b_{11}-\gamma_{2}\right) y_{1}^{2}\right. \\
\left.+\left(\gamma_{3}-\gamma_{1}\right) y_{2}^{2}-\left(b_{33}-\gamma_{2}\right)\right]=0, \\
y_{1}\left[\tilde{b}_{16} y_{1}^{2}+\left(3 \tilde{b}_{26}-2 \tilde{b}_{36}\right) y_{2}^{2}+\tilde{b}_{36}\right]+y_{2}\left[\left(\gamma_{3}-\gamma_{2}\right) y_{1}^{2}\right. \\
\left.\quad+\left(b_{22}-\gamma_{1}\right) y_{2}^{2}-\left(b_{33}-\gamma_{1}\right)\right]=0,
\end{gathered}
$$

where $y_{1}=n_{1} / n_{3}$ and $y_{2}=n_{2} / n_{3}$. The coefficients in (55) and (56) involve with all the prestress components $S_{11}, S_{22}, S_{33}$ and $S_{12}$ present in a monoclinic medium. Thus, if longitudinal wave is not on a co-ordinate plane, then its propagation is affected by all the components of pre-stress present in the medium.

In the absence of pre-stress components, that is, taking $S_{11}=S_{22}=S_{33}=S_{12}=0$, all the expressions derived in this section will reduce to the results which are same as in Ting (2006).

\section{A practical aspect of the problem}

In experiments, when we record the observations related to wave propagation then it is the group velocity (with which energy propagates) that is recorded by the receiver. Ray direction, i.e., the direction between source and receiver is a function of group velocity. So, as far as the practical point of view is concerned, it is the ray direction that can only be compared with the particle motion. Thus, for practical applications, it would be better, if the longitudinal wave could be defined as:

"A wave is said to be longitudinal when the particle motion is parallel to the ray direction."

In this section, the problem of existence of longitudinal waves in pre-stressed anisotropic elastic medium will be discussed using the above definition.

In a spherical co-ordinate system, let $v(\theta, \varphi)$ define the phase velocity of a quasi-wave in a vertical plane along a direction making an angle $\theta$ with the polar (001) axis. Azimuth $\varphi$ measures the orientation of vertical plane from 011-plane towards 101-plane. This defines the phase direction 
$\mathbf{N}=(\sin \theta \cos \varphi, \quad \sin \theta \sin \varphi, \quad \cos \theta)$. Following Ben-Menahem and Sena (1990), the group velocity of a quasi-wave is given by:

$$
\mathbf{w}=v \hat{\mathbf{e}_{r}}+\frac{\partial v}{\partial \theta} \hat{\mathbf{e}_{\theta}}+\frac{1}{\sin \theta} \frac{\partial v}{\partial \varphi} \hat{\mathbf{e}_{\varphi}}
$$

Ray direction or the direction of energy flux of a wave is determined from the components of its group velocity, which are expressed as follows:

$$
\begin{aligned}
& w_{1}=v \cos \varphi \sin \theta+v_{,} \cos \varphi \cos \theta-v_{,_{\varphi}} \frac{\sin \varphi}{\sin \theta}, \\
& w_{2}=v \sin \varphi \sin \theta+v,_{\theta} \sin \varphi \cos \theta-v_{,_{\varphi}} \frac{\cos \varphi}{\sin \theta}, \\
& w_{3}=v \cos \theta+v,_{\theta} \sin \theta
\end{aligned}
$$

where comma before index represents space differentiation.

For a longitudinal wave,

$$
\mathbf{U} \| \mathbf{w} \text {. }
$$

Therefore, equation (1) can be written as:

$$
\left(X_{i k}-h \delta_{i k}\right) w_{k}=0, \quad(i=1,2,3) .
$$

Using the values of $h$ and $X_{i k}$ from (2) and (3), above relation gives three equations, given as:

$$
\begin{aligned}
& \mathbf{N}\left(\mathbf{A}_{1} w_{1}+\mathbf{A}_{4} w_{2}+\mathbf{A}_{5} w_{3}\right) \mathbf{N}^{T}-S_{11} w_{1} \\
& \quad+w_{2}\left(S_{11} n_{1} n_{2}+S_{12} n_{2}^{2}+S_{13} n_{2} n_{3}-S_{12} n_{1}^{2}\right. \\
& \left.\quad-S_{22} n_{1} n_{2}-S_{23} n_{1} n_{3}-S_{12}\right) \\
& \quad+w_{3}\left(S_{11} n_{1} n_{3}+S_{13} n_{3}^{2}+S_{12} n_{2} n_{3}-S_{13} n_{1}^{2}\right. \\
& \left.\quad-S_{33} n_{1} n_{3}-S_{23} n_{1} n_{2}-S_{13}\right)=\left(\rho v^{2}-\mathbf{N S N}^{T}\right) w_{1},
\end{aligned}
$$

$$
\begin{aligned}
& \mathbf{N}\left(\mathbf{A}_{6} w_{1}+\mathbf{A}_{2} w_{2}+\mathbf{A}_{7} w_{3}\right) \mathbf{N}^{T}-S_{22} w_{2} \\
& \quad+w_{1}\left(S_{22} n_{1} n_{2}+S_{12} n_{1}^{2}+S_{23} n_{1} n_{3}-S_{12} n_{2}^{2}\right. \\
& \left.\quad-S_{11} n_{1} n_{2}-S_{13} n_{2} n_{3}-S_{12}\right) \\
& \quad+w_{3}\left(S_{22} n_{2} n_{3}+S_{23} n_{3}^{2}+S_{12} n_{1} n_{3}-S_{23} n_{2}^{2}\right. \\
& \left.\quad-S_{13} n_{1} n_{2}-S_{33} n_{2} n_{3}-S_{23}\right)=\left(\rho v^{2}-\mathbf{N S N}^{T}\right) w_{2},
\end{aligned}
$$

$$
\begin{aligned}
& \mathbf{N}\left(\mathbf{A}_{8} w_{1}+\mathbf{A}_{9} w_{2}+\mathbf{A}_{3} w_{3}\right) \mathbf{N}^{T}-S_{33} w_{3} \\
& \quad+w_{1}\left(S_{33} n_{1} n_{3}+S_{13} n_{1}^{2}+S_{23} n_{1} n_{2}-S_{13} n_{3}^{2}\right. \\
& \left.\quad-S_{11} n_{1} n_{3}-S_{12} n_{2} n_{3}-S_{13}\right) \\
& \quad+w_{2}\left(S_{33} n_{2} n_{3}+S_{23} n_{2}^{2}+S_{33} n_{2} n_{3}-S_{23} n_{3}^{2}\right. \\
& \left.\quad-S_{12} n_{1} n_{3}-S_{22} n_{2} n_{3}-S_{23}\right)=\left(\rho v^{2}-\mathbf{N S N}^{T}\right) w_{3} .
\end{aligned}
$$

Equations (61)-(63) are three non-linear equations in three components $n_{1}, n_{2}, n_{3}$ and hence may not be solved, analytically or numerically. However, the graphical verifications can be made for any given numerical model of a pre-stressed medium. Take a numerical example of Paragneiss, a general anisotropic crystalline rock. The density of the medium is $2727 \mathrm{~kg} / \mathrm{m}^{3}$. The elastic matrix $(\mathrm{GPa})$ for Paragneiss (Rasolofosaon and Zinszner 2002) is given by:

$$
\begin{aligned}
& b_{11}=106.8, \quad b_{12}=27.10, \quad b_{13}=9.68, \\
& b_{14}=-0.03 Z_{1}, b_{15}=0.28 Z_{1}, b_{16}=0.12 Z_{2} ; \\
& b_{22}=99.00, \quad b_{23}=18.22, \quad b_{24}=1.49 Z_{1}, \\
& b_{25}=0.13 Z_{1}, \quad b_{26}=-0.58 Z_{2} ; \\
& b_{33}=54.57, \quad b_{34}=2.44 Z_{1}, \\
& b_{35}=-1.69 Z_{1}, \quad b_{36}=0.75 Z_{2} ; \\
& b_{44}=25.97, \quad b_{45}=1.98 Z_{2}, \quad b_{46}=0.43 Z_{1}, \\
& b_{55}=26.05, \quad b_{56}=1.44 Z_{1}, \quad b_{66}=37.82,
\end{aligned}
$$

where $b_{i j}$ are the components of a square matrix of order six and represent the elastic constant tensor $b_{i j k l}$ in two-suffix notation (Crampin 1989). The assumed values in matrix $\mathbf{S}=\left\{5,0.6 Z_{2}, 0.45 Z_{1}\right.$; $\left.0.6 Z_{2}, 10,0.5 Z_{1} ; 0.45 Z_{2}, 0.5 Z_{1}, 7.5\right\}$, defines the amount (in GPa) of pre-stress in the medium. The values of $Z_{1}=Z_{2}=1$ define the anisotropic medium of arbitrary (triclinic) type. The values $Z_{1}=0, Z_{2}=1$ represent the monoclinic symmetry and $Z_{1}=Z_{2}=0$ represent the orthorhombic symmetry, in the anisotropic elastic medium considered. Define $\psi$ as the angle between group velocity $(\mathbf{w})$ and polarization vector $(\mathbf{U})$. Using the above numerical values, $\psi$ is calculated in the presence of pre-stresses. The phase direction $(\theta, \phi)$ is considered to be varying from $(0,0)$ to $\left(90^{\circ}, 90^{\circ}\right)$. Variations of $\psi$ with phase direction along with the plane $\psi=0$ are plotted in figures $1-3$. Variations in $\psi$ are computed in degrees by the relation

$$
\psi=\cos ^{-1}\left(\frac{\mathbf{U} \cdot \mathbf{W}}{W}\right) .
$$




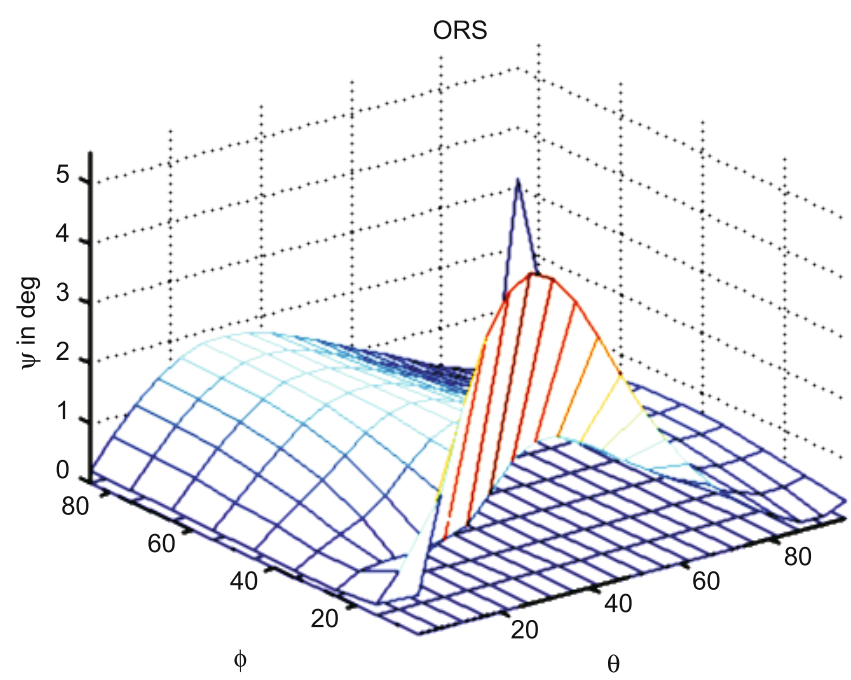

Figure 1. Variations of angle $\psi$ between particle motion $\mathbf{U}$ and ray direction $\mathbf{M}$ along with the plane $\psi=0$, for longitudinal-wave due to pre-stresses, in orthorhombic (ORS) anisotropic Paragneiss rock, with the phase direction $(\theta, \varphi)$, in degrees.

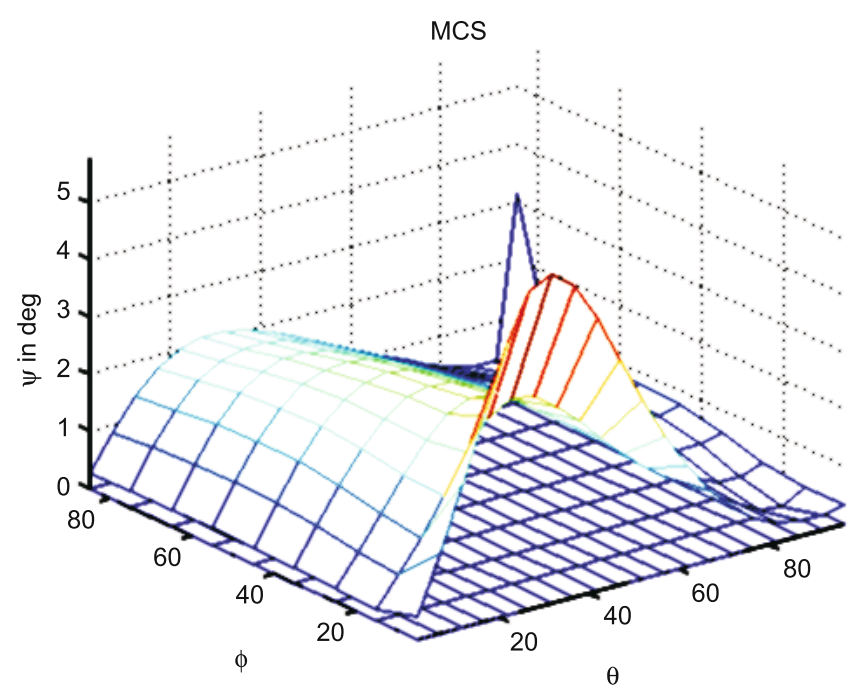

Figure 2. Same as in figure 1, but for monoclinic anisotropic medium.

Directions corresponding to the intersection of graphs of $\psi$ and the plane $\psi=0$ depict the specific directions along which longitudinal waves propagate in pre-stressed anisotropic elastic medium. Details are as follows.

Figure 1 corresponds to the specific directions for the existence of longitudinal waves when the elastic medium is anisotropic with orthorhombic (ORS) symmetry. Figures 2 and 3 correspond to the media with monoclinic (MCS) and triclinic (TCS) symmetry of anisotropy respectively. It is observed from the figures that as the degree of symmetry of planes in the elastic medium decreases, the number of specific directions for the existence of longitudinal waves increases. The deviation of any point

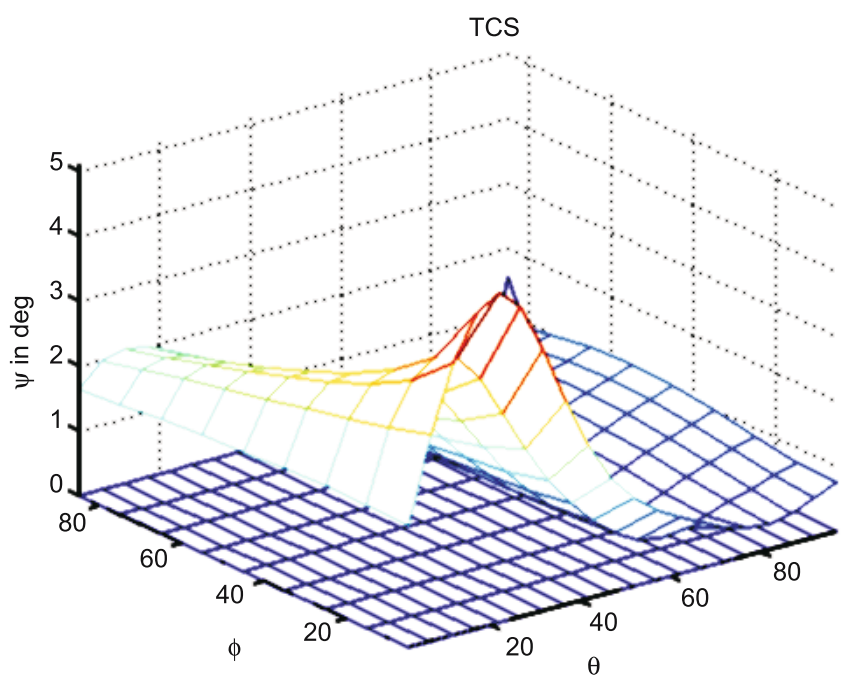

Figure 3. Same as in figure 1, but for triclinic anisotropic medium.

in the graph of $\psi$ from the plane $\psi=0$ gives the angular deviation of particle motion from the ray direction. The kinks in the graph provide the specific directions along which the particle motion is farthest from the ray direction.

If none of the pre-stress components vanishes, then along $x_{1}$-axis $\left(\theta=90^{\circ}, \varphi=0^{\circ}\right), x_{2}$-axis $\left(\theta=90^{\circ}, \varphi=90^{\circ}\right)$ and $x_{3}$-axis $\left(\theta=0^{\circ}, 0^{\circ} \leq \varphi\right.$ $\left.\leq 90^{\circ}\right)$, the longitudinal waves may propagate in orthotropic and monoclinic media. Triclinic medium is the most general type of anisotropy. So, in this medium the behaviour of waves is different from other mediums. In the triclinic medium stressed with all the pre-stress components, longitudinal waves may not propagate along $x_{3}$-axis. In a similar fashion, along $n_{1}=0$ plane $\left(\varphi=90^{\circ}\right)$, $n_{2}=0$ plane $\left(\varphi=0^{\circ}\right)$ and $n_{3}=0$ plane $\left(\theta=90^{\circ}\right)$, longitudinal waves may propagate in orthotropic and monoclinic media. In triclinic medium, longitudinal waves may propagate along $n_{3}=0$ plane but not along $n_{1}=0$ plane and $n_{2}=0$ plane. If any arbitrary direction or plane is chosen then the specific directions for the existence of longitudinal waves can be found by the intersection points of graph for $\psi$ and the plane $\psi=0$.

\section{Conclusions}

In the present work, the analytical expressions for the existence of longitudinal waves in pre-stressed anisotropic elastic medium have been established. These expressions are in terms of phase direction and elastic constants along with pre-stresses present in the medium. A practical aspect to calculate the specific directions for the existence of longitudinal waves has also been suggested. Thus, the existence of longitudinal waves has been 
studied making use of group velocity instead of phase velocity. It is the group velocity but not the phase velocity that is recorded by the receiver. In other words, practically feasible results have been presented. It has been shown that the practical approach is more problematic. This results in non-linear equations which may not be solved analytically or numerically. Only the graphical verifications can be made. The specific directions along which longitudinal waves can propagate in pre-stressed orthotropic, triclinic and monoclinic anisotropic mediums have been shown graphically using a numerical example. This graphical work has presented a clear picture of the problem discussed in the paper. It has been shown that the ray direction and particle motion, which can only be measured at receiver in any geophysical experiment, can provide these specific directions and can be verified graphically only. But if commonly used definition of longitudinal waves in terms of phase propagation direction is used, then the conditions for the existence of longitudinal waves can be established analytically. These conditions are valid but may not be practically applicable in each situation.

In orthotropic medium, longitudinal wave can propagate:

- along the co-ordinate axes without any effect of $S_{11}, S_{22}$ and $S_{33}$.

- along the plane $x_{i}=0(i=1,2,3)$ without any effect of $S_{i i}$, where repeated index does not stand for summation.

- not on any co-ordinate plane and is affected by the pre-stress components present in the medium.

In monoclinic medium, longitudinal wave can propagate:

- along the $x_{1}$-axis and $x_{2}$-axis, if $S_{12}$ vanishes. The wave propagation along these axes is not affected by pre-stress components, $S_{11}, S_{22}$ and $S_{33}$.

- along the $x_{3}$-axis without any effect of prestresses present in the medium.

- along the plane $x_{i}=0(i=1,2,3)$ without any effect of $S_{i i}$, where repeated index does not stand for summation.

- not on any co-ordinate plane and is affected by all the pre-stress components present in the medium.

In triclinic medium, longitudinal wave can propagate:

- along the co-ordinate axis $x_{i}(i=1,2,3)$, if the medium is stressed with $S_{11}, S_{22}, S_{33}$ and $S_{13}$ only. The longitudinal waves propagating along co-ordinate axes are not affected by the pestresses present in the medium.

- along the plane $x_{i}=0(i=1,2,3)$ without any effect of $S_{i i}$, where repeated index does not stand for summation.

- not on any co-ordinate plane and is affected by all the pre-stress components present in the medium.

Various observations made in different media reveal that in all the three mediums, i.e., orthotropic, monoclinic and triclinic media, the longitudinal waves can propagate. These waves may propagate along co-ordinate axes and coordinate planes without any effect of pre-stresses but with condition of having some specific prestress components as zero. These restrictions on pre-stress components differ in different media. The propagation is significantly affected due to prestresses when longitudinal waves propagate not on any co-ordinate plane.

In fact, the earth behaves as a pre-stressed anisotropic model. Thus, the work presented in this paper will help to make the study of the interior of earth better. This study may be useful for the detection of anisotropic materials in earth's crust. The expressions for the existence of longitudinal waves in anisotropic medium are available in literature, but without taking into consideration the pre-stresses present in the medium. So, if the introduction of pre-stresses can provide the expressions for a more realistic model then we should take a step forward in that direction to get more feasible results.

\section{References}

Aleksandrov K S 1956 Propagation of elastic waves along specific directions in crystals; Soviet Physics, Crystallography 1 563-570.

Ben-Menahem A and Sena A G 1990 Seismic source theory in stratified anisotropic media; J. Geophys. Res. 95 $15,395-15,427$.

Biot M A 1939 Non-linear theory of elasticity and the linearized case for a body under initial stress; Phil. Mag. $\mathbf{2 7}$ (7) 468-489.

Biot M A 1940 Influence of initial stress on elastic waves; J. Appl. Phys. 11 522-530.

Biot M A 1965 Mechanics of Incremental Deformation; New York, John Wiley.

Birch F 1947 Finite elastic strain of cubic crystals; Phys. Rev. 71 809-842.

Birch F 1952 Elasticity and constitution of the Earth's interiors; J. Geophys. Res. 57 227-286.

Borgnis F E 1955 Specific directions of longitudinal wave propagation in anisotropic media; Phys. Rev. 98 1000-1005.

Cauchy A L 1827 Exercise de Mathematique; vol. 2, Paris, Bure Feres.

Crampin S 1987 Geological and industrial implications of extensive dilatancy anisotropy; Nature 328 491-496. 
Crampin S 1989 A review of wave motion in anisotropic and cracked elastic media; Wave Motion $\mathbf{3}$ 343-391.

Degtyar A D and Rokhlin S I 1998 Stress effect on boundary conditions and elastic wave propagation through an interface between anisotropic media; J. Acoust. Soc. Am. 104 1992-2003.

Hayes M 1963 Wave propagation and uniqueness in prestressed elastic solids; Proc. Roy. Soc. London A274 500-506.

Kolodner I 1996 Existence of longitudinal waves in anisotropic media; J. Acoust. Soc. Am. 40 730-731.

Man C S and Lu W Y 1987 Towards an acoustoelastic theory for measurements of residual stress; J. Elast. 17 159-182.

Prikazchikov D A and Rogerson G A 2003 Some comments on the dynamic properties of anisotropic and strongly anisotropic pre-stressed elastic solids; Int. J. Engg. Sci. 41 149-171.
Rasolofosaon P N J and Zinszner B E 2002 Comparison between permeability anisotropy and elasticity anisotropy of reservoir rocks; Geophysics 67 230-240.

Sharma M D and Garg N 2006 Wave velocities in a prestressed anisotropic elastic medium; J. Earth System Sci. 115(2) 257-265.

Shuvalov A L 1998 Topological features of the polarization fields of plane acoustic waves in anisotropic media; Proc. Roy. Soc. London A454 2911-2947.

Ting T C T 2006 Longitudinal and transverse waves in anisotropic elastic materials; Acta Mechanica DOI 10.1007/s00707-006-0333-8.

Tolstoy I 1982 On elastic waves in prestressed solid; J. Geophys. Res. 87 6823-6827.

Truesdell C 1952 The mechanical foundations of elasticity and fluid dynamics; J. Ration. Mech. Anal. 1 125-300.

Truesdell C 1966 Existence of longitudinal waves; J. Acoust. Soc. Am. 40 729-730.

MS received 1 August 2008; revised 1 February 2009; accepted 6 June 2009 